\title{
Radar and Radio Signal Processing
}

\author{
John E. Ball ${ }^{*, \dagger}$ and Nicolas H. Younan ${ }^{\dagger}$ \\ Electrical and Computer Engineering, Mississippi State University, 406 Hardy Road, Mississippi State, MS 39762, \\ USA; younan@ece.msstate.edu \\ * Correspondence: jeball@ece.msstate.edu; Tel.: +1-662-325-4169 \\ + These authors contributed equally to this work.
}

Received: 28 August 2017; Accepted: 30 August 2017; Published: 1 September 2017

\section{Introduction}

Radar is a technology used in many aspects of modern life, with many diverse civilian and military applications. Although radars have been around since 1904 [1], much work is still spent today designing, building, testing, and implementing new radars and developing new and more powerful radar signal processing techniques. Radar signal processing is still a very active area of research.

\section{The Special Issue}

This special issue [2] has eight papers covering a diversity of categories in radar signal processing, including three papers on radar optimization and system design [3-5], one paper on parameter estimation [6], three papers in the area of synthetic aperture radar (SAR) and inverse SAR (ISAR) processing [7-9], and one paper on harmonic radar [10].

Two papers were selected as feature papers for the special issue: "Radar Angle of Arrival System Design Optimization Using a Genetic Algorithm" by Egger et al. [3] and "Knowledge-Aided Covariance Matrix Estimation in Spiky Radar Clutter Environments" by Bang et al. [6].

In the category of radar design and optimization, Egger et al. investigated genetic algorithms to aid in the radar design of a multi-beam angle of arrival estimation system [3]. By utilizing properties of Sodoku puzzles, Bufler et al. developed novel waveforms with desirable ambiguity functions and developed methods for antenna array interleaving, thinning, and random element spacing [4]. Pinchera et al. propose a modified generalized alternate projection algorithm which utilizes a simple convex programming implementation to design the element excitations and positions for a sparse array synthesis [5].

In the area of parameter estimation, Bang et al. examined knowledge-aided covariance matrix estimation in complex clutter environments [6].

In the area of SAR processing, Dabbiru et al. utilized textural and wavelet-based features from SAR imagery to detect slump slides in earthen levees [7]. Penner et al. developed a 2D ground-based SAR system and associated 3D time-domain backpropagation algorithm to generate 3D radar images of trees [8]. In ISAR processing, Khwaja et al. propose an orthogonal matching pursuit (OMP) reconstruction algorithm that requires a dictionary based only on first-order phase terms and estimates the second-order and third-order phase terms [9].

By utilizing harmonic radar, where the receive signal is a harmonic of the transmit frequency, Gallagher et al. develop a harmonic radar which has a high clutter rejection capability for moving target imaging and synthetic aperture imaging [10].

Conflicts of Interest: The authors declare no conflict of interest.

\section{References}

1. Hulsmeyer, C. The Telemobiloscope. Electr. Mag. 1904, 2, 388. 
2. Electronics Special Issue Radio and Radar Signal Processing. 2017. Available online: http://www.mdpi. com/journal/electronics/special_issues/radio_radar (accessed on 28 August 2017).

3. Egger, N.; Ball, J.E.; Rogers, J. Radar Angle of Arrival System Design Optimization Using a Genetic Algorithm. Electronics 2017, 6, 24. doi:10.3390/electronics6010024.

4. Bufler, T.D.; Narayanan, R.M.; Sherbondy, K.D. Sudoku inspired designs for radar waveforms and antenna arrays. Electronics 2017, 6, 13. doi:10.3390/electronics6010013.

5. Pinchera, D.; Migliore, M.D.; Lucido, M.; Schettino, F.; Panariello, G. A Compressive-Sensing Inspired Alternate Projection Algorithm for Sparse Array Synthesis. Electronics 2016, 6, 3. doi:10.3390/electronics6010003.

6. Bang, J.H.; Melvin, W.L.; Lanterman, A.D. Knowledge-Aided Covariance Matrix Estimation in Spiky Radar Clutter Environments. Electronics 2017, 6, 20. doi:10.3390/electronics6010020.

7. Dabbiru, L.; Aanstoos, J.V.; Ball, J.E.; Younan, N.H. Screening Mississippi River Levees Using Texture-Based and Polarimetric-Based Features from Synthetic Aperture Radar Data. Electronics 2017, 6, 29. doi:10.3390/electronics6020029.

8. Penner, J.F.; Long, D.G. Ground-Based 3D Radar Imaging of Trees Using a 2D Synthetic Aperture. Electronics 2017, 6, 11. doi:10.3390/electronics6010011.

9. Khwaja, A.S.; Cetin, M. Compressed Sensing ISAR Reconstruction Considering Highly Maneuvering Motion. Electronics 2017, 6, 21. doi:10.3390/electronics6010021.

10. Gallagher, K.A.; Narayanan, R.M.; Mazzaro, G.J.; Martone, A.F.; Sherbondy, K.D. Static and moving target imaging using harmonic radar. Electronics 2017, 6, 30. doi:10.3390/electronics6020030.

(C) 2017 by the authors. Licensee MDPI, Basel, Switzerland. This article is an open access article distributed under the terms and conditions of the Creative Commons Attribution (CC BY) license (http:/ / creativecommons.org/licenses/by/4.0/). 